\title{
Malaria prevalence among patients with acute undifferentiated fever in secondary hospitals in India
}

\author{
Christel Gill Haanshuus ${ }^{*}$, Rosario Vivek², Deepika Xena ${ }^{2}$, Sara Chandy², Dilip Mathai ${ }^{2}$, Anand Manoharan², \\ Nina Langeland ${ }^{3}$, Bjørn Blomberg ${ }^{1,3}$, Kristine Mørch ${ }^{1}$
}

From Challanges in malaria research: Core science and innovation

Oxford, UK. 22-24 September 2014

\section{Background}

The World Health Organization reported approximately one million malaria cases and 500 deaths in India in 2012 based on national surveillance data. Estimated number of deaths range from 20,000 to 200,000 . The aim of this study was to identify the malaria prevalence among patients with acute undifferentiated fever in rural hospitals in India.

\section{Materials and methods}

During April 2011-November 2012, 1564 patients aged $>5$ years were included in a larger fever study from seven secondary hospitals in India located in Assam (Tezpur), Bihar (Raxaul), Chhattisgarh (Mungeli), Maharashtra (Ratnagiri), Andhra Pradesh (Anantapur) and Tamil Nadu (Oddanchatram and Ambur). Routine microscopy, genus-specific mitochondrial PCR and the rapid immunochromatographic test (RDT) Parahit Total $^{\mathrm{TM}}$ (Span Diagnostics Ltd, Surat, India) were performed. Species-specific 18S PCR or sequencing was performed on genus PCR positive samples. Samples with discordancy between PCR and RDT were retested by PCR from the extraction step. PCR was considered as gold standard.

\section{Results}

EDTA blood for PCR was available from 1416 patients and $19 \%$ were malaria PCR positive. The prevalence ranged from lowest $6 \%$ in Oddanchatram (South India) to highest 35\% in Ratnagiri (West India). P. falciparum single infection was detected in $46 \%, 38 \%$ had $P$. vivax, and
11\% mixed infections with $P$. falciparum and $P$. vivax. P. malariae was detected in 5\%. Compared with PCR, the sensitivity of the RDT was $24 \%$ and specificity $99 \%$. The sensitivity of microscopy was $29 \%$ and specificity $98 \%$. A trend of incorrectly diagnosing $P$. falciparum as P. vivax by microscopy was found.

\section{Conclusions}

Our findings support that malaria is an important differential diagnosis in acute undifferentiated fever in all parts of India, and PCR identified a high level of submicroscopic malaria. The very low sensitivity of the RDT emphasizes the importance of choosing a test with high sensitivity, for which WHO's validation of RDTs should be used.

\section{Authors' details}

National Center for Tropical Infectious Diseases, Haukeland University Hospital, Bergen, Norway. ${ }^{2}$ Department of Medicine Unit-1 and Infectious Diseases, Infectious Diseases Training and Research Center, Christian Medical College, Vellore, India. ${ }^{3}$ University of Bergen, Bergen, Norway.

Published: 22 September 2014

doi:10.1186/1475-2875-13-S1-P40

Cite this article as: Haanshuus et al:: Malaria prevalence among patients with acute undifferentiated fever in secondary hospitals in India. Malaria Journal 2014 13(Suppl 1):P40. 